\title{
Evaluation of the RAAPS Risk Screening Tool for Use in Detecting Adolescents With Depression
}

\author{
Jennifer Salerno, DNP, CPNP, FAANP, and Sarah Barnhart, MPH \\ Jennifer Salerno, DNP, CPNP, FAANP, is Director; Sarah Barnhart, MPH, is Research Assistant, University of Michigan, Ann Arbor, MI, USA.
}

\section{Search terms:}

Adolescent depression, depression questionnaires, preventive services, risk

screening questionnaires, risk taking

\author{
Author contact: \\ jsalerno@umich.edu, with a copy to the Editor: \\ kathleen_r_delaney@rush.edu \\ Financial Disclosure: \\ No external funding was secured for this study. \\ Dr. Jennifer Salerno is the President of \\ Possibilities for Change, the company offering \\ license agreements for use of the web-based \\ version of the RAAPS tool. The paper version is \\ available for download at no cost at \\ http://www.raaps.org \\ Sarah Barnhart: No financial disclosures.
}

PROBLEM: Screening is vital to quality adolescent care, but using multiple surveys to screen depression and interrelated risk behaviors is difficult. The aim of this study was to evaluate RAAPS (Rapid Assessment for Adolescent Preventive Services) effectiveness as a screening tool for adolescent depression.

METHODS: Cronbach's $\alpha$, area under the receiver operating characteristic (ROC) curve, sensitivity, specificity, and positive and negative predictive values were assessed.

FINDINGS: Cronbach's $\alpha$ was 0.66 overall. The ROC curve is 0.86 . Sensitivity and specificity were 0.86 and 0.71 , respectively. The positive predictive value is 0.46 and the negative predictive value is 0.95 .

CONCLUSION: Results indicate RAAPS may be an effective screening tool for identifying adolescent depression.

doi: $10.1111 /$ jcap. 12060

\section{Introduction}

Depression is reported to occur in over $26 \%$ of adolescents and can lead to morbidity, mortality, and social problems that last into adulthood (Centers for Disease Control and Prevention, 2010; National Alliance to Advance Adolescent Health, 2011). Depression rates begin increasing in early puberty and continue throughout adolescence, particularly in girls, although rates of completed suicide are higher among boys (Institute of Medicine (U.S.) and National Research Council (U.S.) Committee on the Science of Adolescence, 2011). Furthermore, adolescents often engage in multiple risky behaviors such as substance use, unprotected sexual activities, self-harm, and aggressive behaviors that can have serious effects on their mental health (Institute of Medicine (U.S.) and National Research Council (U.S.) Committee on the Science of Adolescence, 2011). Adolescents with symptoms of depression have been shown to engage in these unsafe behaviors at much higher rates, putting them at even greater risk (Brown et al., 2006; Elkington, Bauermeister, \& Zimmerman, 2010; Waller et al., 2006). It has been noted that $80 \%$ of children do not receive much-needed mental health services and as adults they utilize more healthcare services and have higher healthcare costs than their counterparts who had received services (Kataoka, Zhang, \& Wells, 2002; U.S. Department of Health and Human Services New Freedom Commission on Mental Health, 2003). The Institute of Medicine states that mental health screening offers the potential to intervene early and prevent fully developed disorders (National Research Council (U.S.) and Institute of Medicine (U.S.) Committee on the Prevention of Mental Disorders and Substance Abuse Among Children, Youth, and Young Adults: Research Advances and Promising Interventions, 2009). Moreover, studies have shown that identification of mental health symptoms, improved access to mental health services, and provision of interventions before the onset of fully developed disorders offer the greatest cost-effective approach (National Research Council (U.S.) and Institute of Medicine (U.S.) Committee on the Prevention of Mental Disorders and Substance Abuse Among Children, Youth, and Young Adults: Research Advances and Promising Interventions, 2009; Sturm, 1997).

National organizations have responded by recommending routine screening in primary care settings of adolescents for all risky behaviors including depression screening (Centers for Disease Control and Prevention, 2010; Maternal Child 
Health Bureau: Bright Futures, 2012; U.S. Preventive Services Task Force, 2012). An assessment of adolescent depression and contributing risky behaviors is vital in providing quality adolescent care, but using multiple surveys to provide effective screening is difficult and time consuming. In primary care settings, provision of adolescent preventive services that include screening for depression remains far below recommended levels (Halpern-Felsher et al., 2000; Irwin, Adams, Park, \& Newacheck, 2009; Ma, Wang, \& Stafford, 2005; Rand, Auinger, Klein, \& Weitzman, 2005), with time noted as one of the biggest barriers (Cheng, DeWitt, Savageau, \& O'Connor, 1999). Hence, a comprehensive, self-administered adolescent risk screening tool that has been validated to identify depressive symptoms is an attractive alternative. The American Academy of Pediatrics Task Force on Mental Health recommends primary care practices institute routine, periodic screening using validated instruments to identify adolescents with mental health symptoms (Foy, Kelleher, Laraque, \& American Academy of Pediatrics Task Force on Mental Health, 2010). Universal depression screening for adolescents has been met with little resistance and has been well received and accepted by primary care providers (Zuckerbrot et al., 2007).

\section{Methods}

The aim of this study was to evaluate the criterion validity and internal reliability of the Rapid Assessment for Adolescent Preventive Services (RAAPS) as a screening tool for adolescent depression, thereby extending the use of the RAAPS to settings interested in both identifying adolescent depression and comorbid risky behaviors that may negatively impact mental health. The RAAPS is a 21-item adolescent risk screening tool developed to identify the risk behaviors contributing most to adolescent morbidity, mortality, and social problems (Yi, Martyn, Salerno, \& Darling-Fisher, 2009). The RAAPS was developed to overcome barriers to risk screening, particularly the time it takes to administer and review standardized risk screening tools in clinical practice settings. The RAAPS has been recognized as a valid, time-efficient, and effective screening tool in identifying multiple risk behaviors in adolescents, with strengths reported as its concise format, ease of use, and ability to assess for major risks efficiently (Yi et al., 2009; Salerno, Marshall, \& Picken, 2012). Face-, content-, and criterion-related validity and inter-rater and equivalence reliability for the RAAPS were established in a previous study (Salerno et al., 2012). This study focused specifically on the validity and internal reliability of the RAAPS as a tool to identify adolescent depression.

During the development of the RAAPS screening tool, recommendations from national health organizations, relevant literature, and adolescent risk behavior statistics were reviewed to ensure the risk behaviors contributing most to adolescent morbidity and mortality were targeted for inclusion. The RAAPS content areas include eating/weight, physical activity, unintentional injury/violence, substance use, sexual health, depression/self-harm, and adult support. Specific questions targeted to identify those youths at greatest risk for clinical depression include content such as experiences of being bullied, feelings of sadness and anxiety, suicidal thoughts or actions, and self-harming behaviors such as cutting. Pertinent content was combined to obtain the most information with the fewest number of questions. The RAAPS screening tool is copyrighted by the University of Michigan, and therefore was not published within this article and can be reviewed and downloaded for use free of charge at http://www.raaps.org.

This is an expansion of investigation of reliability in which we hypothesized that the RAAPS could yield results similar to the Patient Health Questionnaire-Adolescent (PHQ-A) in identifying depression symptoms in adolescents while simultaneously identifying additional risk behaviors that may contribute to their depressed feelings and therefore be more clinically useful as an initial comprehensive screening tool. A comprehensive, self-administered adolescent risk screening tool that has been validated to identify depressive symptoms is an attractive clinical alternative to administering multiple tools or using a tool that only screens for depression and no other contributing behaviors. The PHQ is viewed as a "gold standard" for depression screening in primary care settings and has shown to be effective in the identification of adolescent depression requiring follow-up. The PHQ is a brief, well-validated tool measuring depression, anxiety, and somatization (Kroenke, Spitzer, Williams, \& Lowe, 2010). The PHQ-A has been tested for use in adolescents and offers an acceptable tool for early detection and recognition of mental health among adolescents (Johnson, Harris, Spitzer, \& Williams, 2002). In addition, the U.S. Preventative Services Task Force found that the PHQ-A demonstrated good sensitivity and specificity in screening for adolescent depression (U.S. Preventive Services Task Force, 2009).

This study used psychometric methods to establish criterion validity and internal reliability of the RAAPS. Institutional review board approval of the study (HUM00053854) was obtained through the University of Michigan. For this cross-sectional study, we retrospectively reviewed the dataset from a comprehensive adolescent risk questionnaire including both the RAAPS and the PHQ-A questions. This risk questionnaire was part of a more comprehensive health needs assessment given to high school adolescents aged $14-18$ years ( $n=286$ ). Adolescents completing the questionnaire were from the same high school, were general education students and their participation was based on their attendance in classrooms that were participating in the assessment as determined by school administration. Classroom participation 
Table 1. Demographic Characteristics of the Study Population

\begin{tabular}{lc}
\hline Characteristic & $n(\%)$ \\
\hline Age (year) & \\
14 & $24(8.4)$ \\
15 & $79(27.6)$ \\
16 & $92(32.2)$ \\
17 & $87(30.4)$ \\
18 or older & $2(0.7)$ \\
Missing & $2(0.7)$ \\
Gender & \\
Male & $139(48.6)$ \\
Female & $145(50.7)$ \\
Missing & $2(0.7)$ \\
Race/ethnicity & \\
White & $139(49.1)$ \\
African American & $74(26.2)$ \\
Hispanic & $15(5.3)$ \\
Multiracial & $37(13.1)$ \\
Other & $21(7.4)$ \\
Lives primarily with & \\
Mother and father & $131(45.8)$ \\
Mother and father share custody & $23(8.0)$ \\
Mother only & $51(17.8)$ \\
Father only & $11(3.9)$ \\
One parent and stepparent & $43(15.0)$ \\
Other & $24(8.4)$ \\
Missing & $3(1.1)$ \\
\hline
\end{tabular}

was determined based on staff willingness to forgo teaching on the day of administration and to administer the survey to their students instead. An opt-out procedure was used allowing parents the opportunity to decline their children's participation. Adolescents were able to opt out of completing the survey on the day of administration. Demographic data were collected on all participants. A total of 286 adolescents anonymously completed the needs assessment questions. The majority of participants were between the ages of 15 and 17 years. The sample included roughly equal numbers of men and women. White and African American adolescents represented the largest racial groups in this sample. Only $46.3 \%$ of participants lived with both parents. Demographic data are displayed in Table 1.

The dataset had been electronically tallied prior to this study from completed scantrons and was analyzed in this study using Stata 10 (Stata Corp, College Station, TX, USA). Cronbach's $\alpha$, the area under the receiver operating characteristic (ROC) curve, sensitivity, specificity, and positive and negative predictive values were assessed. The RAAPS includes four questions the researchers hypothesized to be related to depression and were included in the analyses:

- During the past month, have you been threatened, teased, or hurt by someone (on the web, by text, or in person), or has anyone made you feel sad, unsafe, or afraid?

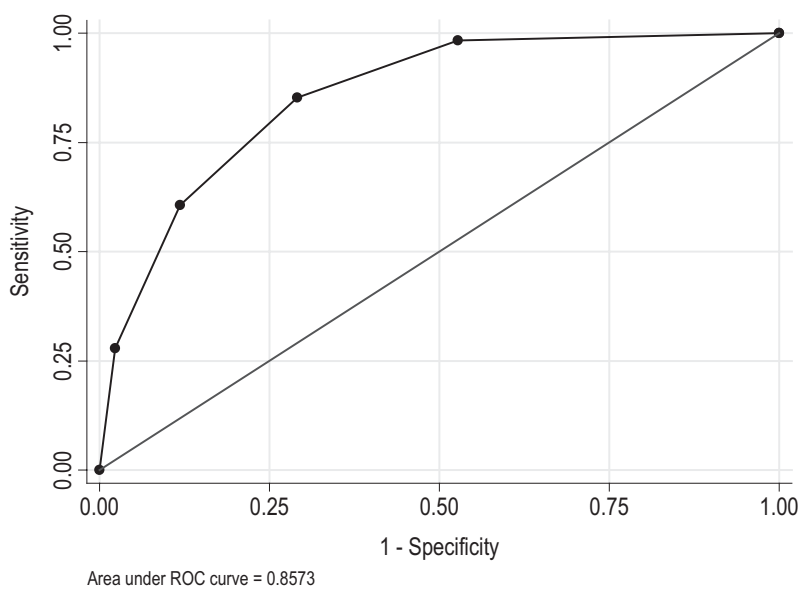

Figure 1. Receiver Operating Characteristic (ROC) Curve

- During the past month, did you often feel very sad or down as though you had nothing to look forward to?

- Do you have any serious problems or worries at home or at school?

- In the past 12 months, have you seriously thought about killing yourself, tried to kill yourself, or have you purposely cut, burned, or otherwise hurt yourself?

PHQ-A scores of 11 or higher, the published cut point for identifying depression, were used to evaluate the sensitivity and specificity of the RAAPS depression screening questions noted above. A ROC curve was used to determine the overall accuracy of the RAAPS depression screening questions. Cronbach's $\alpha$ was calculated to determine the internal reliability of the RAAPS.

\section{Results}

Based on the PHQ-A cut point of a score of 11 or higher, the true prevalence of depression risk was $22.0 \%$ (95\% CI, $17.2-$ 26.9). Cronbach's $\alpha$ demonstrated that all four questions had good internal consistency. The overall $\alpha$ was fair (0.66). The ROC curve demonstrated good accuracy (Figure 1). The area under the curve was 0.86 (95\% CI, 0.81-0.90). The optimal cut point was determined to be two or more positive responses on the RAAPS questions based on the ROC curve. The number of affirmative responses to RAAPS questions is displayed in Table 2. The overall sensitivity was $85.7 \%$ (95\% CI, 74.8-92.5) and the specificity was 71.3\% (95\% CI, 65.076.9). Sensitivity and specificity for individual questions on RAAPS are displayed in Table 3 . The positive predictive value is $45.8 \%(95 \% \mathrm{CI}, 37.1-54.7)$ and the negative predictive value is $94.6 \%$ (95\% CI, 90.0-97.3). Based on a cut point of two or more positive responses on the selected RAAPS items, the prevalence of depression risk was $41.3 \%$ (95\% CI, $35.7-$ 47.0). 
Table 2. Affirmative Responses

\begin{tabular}{lc}
\hline RAAPS questions & $n(\%)$ \\
\hline $\begin{array}{l}\text { During the past month, have you been threatened, } \\
\text { teased, or hurt by someone (on the Internet, by text, }\end{array}$ & $83(29.0)$ \\
or in person), or has anyone made you feel sad, & \\
unsafe, or afraid? & \\
In the past 12 months, have you seriously thought about & $79(27.6)$ \\
$\quad$ killing yourself, tried to kill yourself, or have you & \\
$\quad$ purposely cut, burned, or otherwise hurt yourself? & \\
During the past month, did you often feel very sad or \\
$\quad$ down, as though you had nothing to look forward to? & 131 (45.8) \\
$\begin{array}{l}\text { Do you have any serious problems or worries at home or } \\
\text { school? }\end{array}$ & $88(30.8)$ \\
\hline
\end{tabular}

RAAPS, Rapid Assessment for Adolescent Preventive Services.

Table 3. Sensitivity and Specificity

\begin{tabular}{lll}
\hline RAAPS questions & $\begin{array}{l}\text { Sensitivity } \\
\text { (\%) }\end{array}$ & $\begin{array}{l}\text { Specificity } \\
\text { (\%) }\end{array}$ \\
\hline $\begin{array}{l}\text { During the past month, have you been } \\
\text { threatened, teased, or hurt by someone } \\
\text { (on the Internet, by text, or in person), } \\
\text { or has anyone made you feel sad, unsafe, } \\
\quad \text { or afraid? }\end{array}$ & 55.6 & 78.3 \\
$\begin{array}{l}\text { In the past } 12 \text { months, have you seriously } \\
\text { thought about killing yourself, tried to kill } \\
\quad \text { yourself, or have you purposely cut, } \\
\text { burned, or otherwise hurt yourself? }\end{array}$ & 68.3 & 83.9 \\
$\begin{array}{l}\text { During the past month, did you often feel } \\
\text { very sad or down, as though you had } \\
\text { nothing to look forward to? }\end{array}$ & 85.4 & 64.5 \\
$\begin{array}{l}\text { Do you have any serious problems or worries } \\
\text { at home or school? }\end{array}$ & 63.5 & 78.2 \\
\hline
\end{tabular}

RAAPS, Rapid Assessment for Adolescent Preventive Services.

\section{Discussion}

The RAAPS risk screening tool was designed as a brief, timeefficient tool for use in identifying the risk behaviors contributing most to morbidity, mortality, and social problems in adolescents, and is intended to be used in a variety of settings serving adolescents (Salerno et al., 2012). In a previous study, the RAAPS proved to be a valid and reliable tool for screening risk behaviors contributing most to morbidity and mortality (Salerno et al., 2012). The RAAPS creates a unique opportunity for professionals to meet adolescent risk screening recommendations in a time-efficient and effective manner with less disruption in their organization's work flow.

This study used psychometric methods to evaluate the RAAPS as an effective screening tool to identify depressive symptoms in adolescents that would warrant further assessment. The Cronbach's $\alpha$ analysis of the RAAPS depression screening questions demonstrated fair internal consistency at 0.66 , and 0.7 is considered the cutoff for being acceptable
(Nunnally, 1978); however, this was expected due to the small number of RAAPS depression screening questions in the analysis. Therefore, the lower $\alpha$ value should not be interpreted as strong evidence against internal consistency, and for a behavioral measure 0.66 could be considered reasonable. The ROC curve analysis revealed that the overall accuracy of the RAAPS depression screening questions is acceptable within our study population.

The cut point of two or more positive responses on the RAAPS depression screening questions provides a relatively high degree of sensitivity and specificity. We prioritized higher sensitivity over specificity due to the importance of detecting high-risk mental health behaviors in adolescents and the low risks associated with detecting false positives. Additional screening for depression and/or brief assessment would be recommended for those adolescents with two or more positive responses to the RAAPS depression screening questions to determine whether a more comprehensive depression assessment with a treatment plan and referrals are appropriate.

This study represents preliminary findings of the RAAPS as an adolescent depression screening tool. Validity and reliability of the RAAPS as a measure of adolescent depression were established. With multiple competing demands on health professionals, the RAAPS can assist adolescent serving professionals in identifying depressive symptoms that may require further assessment, treatment, and referral while simultaneously identifying interrelated risk behaviors such as substance use or unsafe sexual activities. Data obtained from quality tools can contribute to the efficient and appropriate use of limited resources and the eventual reduction in health risk behaviors (Kann, Brener, Warren, Collins, \& Giovino, 2002).

As this is a preliminary study, the limitations include the small sample size and limited diversity of the participants. Another important limitation of this study is that the PHQ-A was used as the gold standard for depression screening in adolescents. Although the PHQ-A has been validated as an effective screening tool for depression in the adolescent population, the true gold standard is clinician diagnosis.

\section{Conclusion}

Assessment of adolescent risk that includes depression is vital to providing quality health care to adolescents. However, facilitating multiple surveys necessary to provide recommended comprehensive risk screening is difficult. These preliminary findings indicate the RAAPS is a valid tool for identifying depressive symptoms in adolescents requiring further evaluation, and for identifying other risk behaviors (e.g., substance use, sexual activity, violence) that negatively impact their mental health. The RAAPS has clinical implications for identifying and incorporating into practice an efficient and effective screening instrument for early identifi- 
cation of depression and interrelated risky behaviors impacting adolescent mental health. Early identification allows health professionals the opportunity to provide adolescents with appropriate risk reduction counseling, further assessment, and resources that may positively impact their mental health. Primary mental health care is an essential role of the psychiatric mental health nurse practitioner (PMHNP). Although most PMHNPs work in psychiatric settings, primary care is evolving toward the integration of physical and mental health care, with PMHNPs beginning to provide mental health intervention in primary care settings. The RAAPS would be a useful initial screening tool for the PMHNP providing primary care to identify adolescents requiring a more comprehensive evaluation as well as identify concurrent risky behaviors affecting their mental health.

Future research should focus on examining the RAAPS clinical impact on identifying depression and interrelated risk behaviors in adolescents with a larger, more diverse adolescent sample. Furthermore, process evaluation should be conducted on implementation of RAAPS into a primary care practice setting and examination of the effects on health professional time, patient/provider interaction, and the influence on positive behavior changes over time in adolescent patients.

\section{References}

Brown, L. K., Tolou-Shams, M., Lescano, C., Houck, C., Zeidman, J., Pugatch, D., \& Lourie, K. J. (2006). Depressive symptoms as a predictor of sexual risk among African American adolescents and young adults. Journal of Adolescent Health, 39, 444-e1.

Centers for Disease Control and Prevention. (2010). Youth risk behavior surveillance-United States, 2009. MMWR. Surveillance Summaries: Morbidity and Mortality Weekly Report. Surveillance Summaries, 59, 1-142.

Cheng, T. L., DeWitt, T. G., Savageau, J. A., \& O’Connor, K. G. (1999). Determinants of counseling in primary care pediatric practice: Physician attitudes about time, money, and health issues. Archives of Pediatrics and Adolescent Medicine, 153, 629-635.

Elkington, K. S., Bauermeister, J. A., \& Zimmerman, M. A. (2010). Psychological distress, substance use, and HIV/STI risk behaviors among youth. Journal of Youth and Adolescence, 39(5), 514-527.

Foy, J. M., Kelleher, K. J., Laraque, D., \& American Academy of Pediatrics Task Force on Mental Health. (2010). Enhancing pediatric mental health care: Strategies for preparing a primary care practice. Pediatrics, 125(Suppl. 3), S87-108.

Halpern-Felsher, B. L., Ozer, E. M., Millstein, S. G., Wibbelsman, C. J., Fuster, C. D., Elster, A. B., \& Irwin, C. E., Jr. (2000). Preventive services in a health maintenance organization: How well do pediatricians screen and educate adolescent patients? Archives of Pediatrics and Adolescent Medicine, 154, 173-179.
Institute of Medicine (U.S.) and National Research Council (U.S.) Committee on the Science of Adolescence. (2011). The science of adolescent risk-taking workshop report. Washington, DC: The National Academies Press.

Irwin, C. E., Jr, Adams, S. H., Park, M. J., \& Newacheck, P. W. (2009). Preventive care for adolescents: Few get visits and fewer get services. Pediatrics, 123, e565-e572.

Johnson, J. G., Harris, E. S., Spitzer, R. L., \& Williams, J. B. (2002). The patient health questionnaire for adolescents: Validation of an instrument for the assessment of mental disorders among adolescent primary care patients. Journal of Adolescent Health, 30, 196-204.

Kann, L., Brener, N. D., Warren, C. W., Collins, J. L., \& Giovino, G. A. (2002). An assessment of the effect of data collection setting on the prevalence of health risk behaviors among adolescents. Journal of Adolescent Health, 31, 327-335.

Kataoka, S. H., Zhang, L., \& Wells, K. B. (2002). Unmet need for mental health care among U.S. children: Variation by ethnicity and insurance status. American Journal of Psychiatry, 159, 1548-1555.

Kroenke, K., Spitzer, R. L., Williams, J. B., \& Lowe, B. (2010). The Patient Health Questionnaire Somatic, Anxiety, and Depressive Symptom Scales: A systematic review. General Hospital Psychiatry, 32, 345-359.

Ma, J., Wang, Y., \& Stafford, R. S. (2005). U.S. adolescents receive suboptimal preventive counseling during ambulatory care. Journal of Adolescent Health, 36, 441e1-441e7.

Maternal Child Health Bureau: Bright Futures. (2012). Guidelines for health supervision of infants, children and adolescents. Retrieved from http://brightfutures.aap.org/pdfs/Guidelines _PDF/18-Adolescence.pdf

National Alliance to Advance Adolescent Health. (2011). Significant multiple risk behaviors among U.S. high school students: Fact sheet No. 8. Retrieved from http://www .thenationalalliance.org/pdfs/FS8.\%20Significant\% 20Multiple\%20Risk\%20Behaviors.pdf

National Research Council (U.S.) and Institute of Medicine (U.S.) Committee on the Prevention of Mental Disorders and Substance Abuse Among Children, Youth, and Young Adults: Research Advances and Promising Interventions. (2009). Preventing mental, emotional, and behavioral disorders among young people: Progress and possibilities. Washington, DC: National Academies Press.

Nunnally, J. C. (1978). Psychometric theory (2nd ed.). New York: McGraw Hill.

Rand, C. M., Auinger, P., Klein, J. D., \& Weitzman, M. (2005). Preventive counseling at adolescent ambulatory visits. Journal of Adolescent Health, 37, 87-93.

Salerno, J., Marshall, V., \& Picken, E. (2012). Rapid assessment for adolescent preventive services: Validity and reliability of the RAAPS adolescent risk screening tool. Manuscript accepted Journal of Adolescent Health, 50(6), 595-599.

Sturm, R. (1997). How expensive is unlimited mental health care coverage under managed care? Journal of the American Medical Association, 278, 1533-1537. 
U.S. Department of Health and Human Services New Freedom Commission on Mental Health. (2003). Achieving the promise: Final report. Department of Health and Human Services. No. SMA-03-3832.

U.S. Preventive Services Task Force. (2009). Screening and treatment for major depressive disorder in children and adolescents: US Preventive Services Task Force recommendation statement. Pediatrics, 123, 1223-1228.

U.S. Preventive Services Task Force. (2012, January 6). Major depressive disorder in children and adolescents. Retrieved from http://www.uspreventiveservicestaskforce.org/uspstf/ uspschdepr.htm

Waller, M. W., Hallfors, D. D., Halpern, C. T., Iritani, B. J., Ford, C. A., \& Guo, G. (2006). Gender differences in associations between depressive symptoms and patterns of substance use and risky sexual behavior among a nationally representative sample of U.S. adolescents. Archives of Women's Mental Health, 9(3), 139-150.

Yi, C. H., Martyn, K., Salerno, J., \& Darling-Fisher, C. S. (2009). Development and clinical use of Rapid Assessment for Adolescent Preventive Services (RAAPS) questionnaire in school-based health centers. Journal of Pediatric Health Care, 23, 2-9.

Zuckerbrot, R. A., Maxon, L., Pagar, D., Davies, M., Fisher, P. W., \& Shaffer, D. (2007). Adolescent depression screening in primary care: Feasibility and acceptability. Pediatrics, 119, 101-108. 